\title{
Scintigraphic evidence for cardiac sympathetic dysinnervation in long-term IDDM patients with and without ECG-based autonomic neuropathy
}

\author{
O. Schnell ${ }^{1}$, C.-M. Kirsch ${ }^{2}$, J. Stemplinger ${ }^{1}$, M. Haslbeck ${ }^{1}$, E. Standl ${ }^{1}$ \\ ${ }^{1}$ Diabetes Research Institute, Munich and the Third Medical Department, Schwabing City Hospital, Munich, Germany \\ ${ }^{2}$ Department of Nuclear Medicine, University of Munich, Germany
}

Summary To analyse the presence and extent of global and regional distributions of cardiac sympathetic dysinnervation in long-term insulin-dependent diabetes mellitus (IDDM) without myocardial perfusion abnormalities $\left({ }^{99} \mathrm{~m}\right.$ Tc-methoxy isobutyl isonitrile study), ${ }^{123} \mathrm{I}$-metaiodobenzylguanidine ( $\left.{ }^{123} \mathrm{I}-\mathrm{MIBG}\right)$ scintigraphy was performed in two clinically-comparable groups (20 diabetic patients with and 22 diabetic patients without ECG-based cardiac autonomic neuropathy). For comparison nine control subjects without heart disease were investigated. Only six diabetic patients $(27 \%)$ without and one diabetic patient $(5 \%)$ with ECG-based autonomic neuropathy were found to have a uniform homogeneous uptake of ${ }^{123}$ I-MIBG, in contrast to a uniform homogeneous uptake in all control subjects. The uptake of ${ }^{123}$ I-MIBG in the posterior myocardium of diabetic patients was smaller than in the anterior, lateral and septal myocardium $(p<0.001, p<0.001, p=0.001)$. In addition, diabetic patients with cardiac autonomic neuropathy ( $\geq$ two of five age-related cardiac reflex tests abnormal) demonstrated a more reduced uptake in the global, lateral and posterior myocardium than diabetic patients without $(p<0.01, p<0.01$, $p<0.001$ ). A correlation between global or regional myocardial ${ }^{123} \mathrm{I}-\mathrm{MIBG}$ uptake, however, and duration of diabetes, $\mathrm{HbA}_{1}$, body mass index or QT interval length was not observed. Our study demonstrates that cardiac sympathetic dysinnervation is common in long-term IDDM even in patients without ECGbased cardiac autonomic neuropathy and that the posterior myocardium is predominantly affected. We conclude that ${ }^{123} \mathrm{I}-\mathrm{MIBG}$ scintigraphy is a promising approach to further elucidate the pattern and natural history of myocardial dysinnervation in IDDM. [Diabetologia (1995) 38: 1345-1352]

Key words Insulin-dependent diabetes mellitus, metaiodobenzylguanidine, autonomic neuropathy, sympathetic dysinnervation, QT interval.
Cardiac autonomic neuropathy is associated with an increased risk of morbidity and mortality $[1,2]$. Its clinical evaluation and diagnosis in the last two decades has been mainly based on conventional non-invasive cardiac reflex tests, including measurements of

Received: 8 March 1995 and in revised form: 12 May 1995

Corresponding author: Dr. O. Schnell, Diabetes Research Institute, Kölner Platz 1, D-80804 Munich, Germany

Abbreviations: CAN-negative, Without cardiac autonomic neuropathy; CAN-positive, with cardiac autonomic neuropathy; MIBG, metaiodobenzylguanidine; SPECT, single-photon emission computed tomography; MU, myocardial uptake; ${ }^{99} \mathrm{~m}$ Tc-MIBI, ${ }^{99 \mathrm{~m}} \mathrm{Tc}-\mathrm{methoxy}$ isobutyl isonitrile. heart rate variation at rest and during deep breathing, heart rate response to standing, Valsalva ratio and systolic blood pressure changes to standing [3]. Recently QT interval lengthening has been suggested to be associated with an increased risk of unexpected death in diabetes mellitus $[4,5]$. Cardiac reflex tests and QT interval, however, only indirectly assess cardiac autonomic nerve function in diabetes mellitus. Due to prior lack of techniques to directly investigate damage to the sympathetic neurons in the living heart the pathophysiology of cardiac sympathetic dysinnervation has remained largely unknown.

Single-photon emission computed tomography (SPECT) using the tracer ${ }^{123}$ I-metaiodobenzylguanidine ( ${ }^{123}$ I-MIBG) has been introduced as a new ap- 
Table 1. Clinical characteristics of IDDM patients with or without cardiac autonomic neuropathy and control subjects

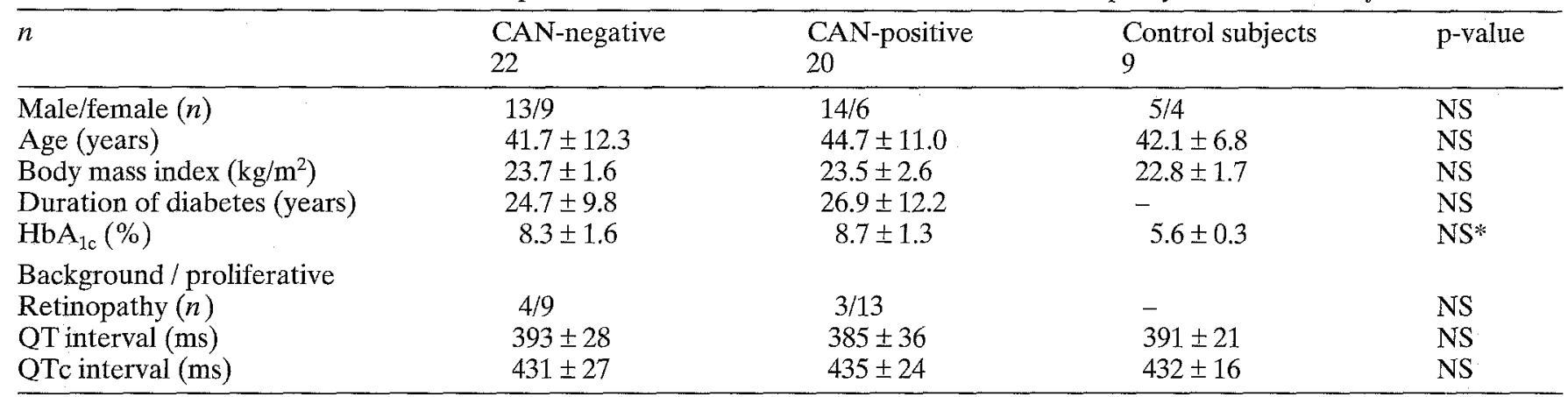

Values are means \pm SD; NS, Not significant; NS*, not significant between the CAN-negative and CAN-positive group; CAN-negative, without cardiac autonomic neuropathy; CAN-positive, with cardiac autonomic neuropathy

proach to directly assess the efferent cardiac sympathetic nervous system $[6,7]$. The guanethidine analogue metaiodobenzylguanidine (MIBG) shares the same active uptake, storage, and release mechanisms as norepinephrine and therefore has a high affinity to chromaffin tissue and adrenergic nerve terminals $[8,9]$.

Abnormal ${ }^{123} \mathrm{I}-\mathrm{MIBG}$ uptake indicating cardiac sympathetic dysinnervation has been demonstrated after myocardial infarction [10], in cardiomyopathy [11] and in the idiopathic long QT syndrome [12]. Pilot studies in patients with diabetes suggest that the myocardial ${ }^{123}$ I-MIBG uptake is reduced in diabetic patients with cardiac autonomic neuropathy [13-15].

The purpose of this study was to 1) determine the pattern of global and regional sympathetic cardiac innervation in long-term IDDM patients with and without ECG-based cardiac autonomic neuropathy and 2) compare the findings with conventional cardiac reflex tests and QT interval.

\section{Subjects and methods}

We examined 22 long-term IDDM patients without ECGbased cardiac autonomic neuropathy (see below) and 20 longterm IDDM patients with ECG-based cardiac autonomic neuropathy. The two groups showed no significant differences in regard to gender, age, body mass index, duration of diabetes, $\mathrm{HbA}_{1}$, presence of diabetic retinopathy, QT and QTc interval (Table 1). All patients were on intensive insulin therapy, which included four or more subcutaneous insulin injections daily or insulin administration by an external pump. Patients with a history of coronary heart disease, myocardial infarction and arrhythmias or cardiac autonomic neuropathy other than of diabetic origin were excluded from the study. Hypoglycaemia $24 \mathrm{~h}$ prior to the study also led to exclusion from the study.

As a control group nine non-diabetic subjects without any evidence of heart disease affecting the myocardial sympathetic nervous system were investigated (Table 1 ). They underwent SPECT using ${ }^{123}$ I-MIBG for exclusion of pheochromocytoma. No patient had documented arrhythmias or symptoms suggestive of arrhythmias.

The diabetic patients and the control subjects were taking no medication known to interfere with ${ }^{123}$ I-MIBG uptake, such as calcium channel blocking agents, beta agonist drugs or tranquilizing agents [16].

${ }^{123}$ I-MIBG study. Preprandial morning blood glucose level was assessed at 07.00 hours and the patients continued their regular morning insulin dose and breakfast before the study. They received $300 \mathrm{mg}$ perchlorate to block thyroid uptake of the tracer. At 09.00 hours a dose of $220 \mathrm{MBq}$ of commercially available ${ }^{123}$ I-MIBG (Medgenix, Brussels, Belgium) was injected as an intravenous bolus into the antecubital vein. At calibration date the radioactive concentration was $74 \mathrm{MBq} / \mathrm{ml}$, the specific activity $148 \mathrm{MBq} / \mathrm{mg}$ and the radiochemical purity $<3 \%$ free iodine.

The homogeneity of ${ }^{123}$ I-MIBG uptake was assessed by a semiquantitative approach. To allow clearance from extraneuronal tissue uptake SPECT images were obtained after $5 \mathrm{~h}$ using a two-headed gamma camera (ROTA 2, Siemens Medical Systems), LEAP collimator, $360^{\circ}$ rotation, and a $64 \times 64$ matrix. Oblique frontal and oblique sagittal slices were obtained. The myocardial ${ }^{123}$ I-MIBG uptake was displayed as polar coordinate maps of relative tracer activity and the maps were divided into four regions (Fig. 1).

Within the global, anterior, lateral, posterior and septal myocardium the mean tracer activity was visually assessed by two independent observers, who were unaware of the patients' history. The findings were grouped into a myocardial uptake score (MU score): 1 = normal homogeneous uptake; 2 = slight reduction of uptake; $3=$ moderate reduction of uptake; $4=$ considerable reduction of uptake; $5=$ severe reduction of uptake; $6=$ no uptake.

An MU score of more than 2 was considered to be abnormal.

${ }^{99 m} T c-M I B I$ study. To ensure that no perfusion abnormalities influenced the MIBG uptake, conventional myocardial scintigraphy was added to the protocol. After the MIBG study the resting patients were injected with $400 \mathrm{MBq}{ }^{99} \mathrm{mc}$-methoxy isobutyl isonitrile ( ${ }^{99}$ Tc-MIBI). This radiopharmaceutical is readily taken up by the myocardium and remains within the myocardial cell depending on its oxidative metabolism [17, $18,19]$. Thus, this examination provides information on myocardial perfusion at rest as well as on metabolic disturbances. Imaging commences $1 \mathrm{~h}$ after the injection to avoid superimposition of liver uptake. The imaging technique applied is identical to that of MIBG. The scintigrams were scored with the same scheme as the MIBG scans.

Cardiac reflex tests. Five cardiac reflex tests were performed in diabetic patients to test for cardiac autonomic neuropathy: 


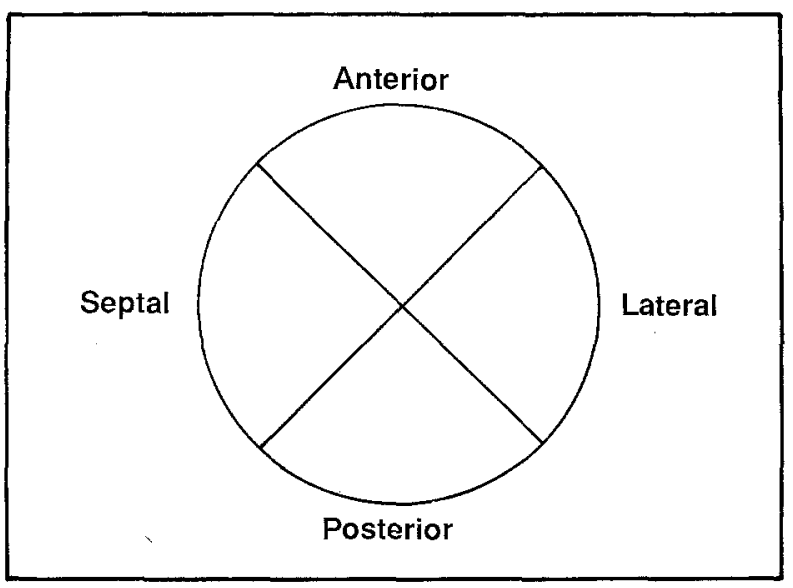

Fig. 1. Scheme of a polar map of myocardial ${ }^{123} \mathrm{I}-\mathrm{MIBG}$ uptake. Four different myocardial regions (anterior, lateral, posterior and septal) were evaluated

1) recording of heart rate variation at rest over 170 consecutive beats and calculation of the coefficient of variation. 2) Heart rate variation during deep breathing at six breaths per min with calculation of the ratio of the longest RR-interval during expiration and the shortest RR-interval during inspiration. 3) Immediate heart rate response to standing expressed by the $30: 15$ ratio. 4) Valsalva manoeuvre and calculation of the ratio of the longest RR-interval after and the shortest RR-interval during the manoeuvre. 5) Difference between the lying and standing systolic blood pressure.

Heart rate variation at rest and deep breathing, heart rate response to standing and the Valsalva ratio were performed with the ProSciCard computer system (MediSyst, Linden, Germany). For the evaluation age-related normal ranges were used [20]. Criterion for an abnormal blood pressure response was a systolic blood pressure drop on standing of $\geq 30 \mathrm{mmHg}$. Each diabetic patient was classified according to the number of abnormal cardiac reflex tests: $<2$ cardiac reflex tests $=$ no cardiac autonomic neuropathy (CAN-negative) $\geq 2$ abnormal reflex tests $=$ cardiac autonomic neuropathy (CAN-positive).

$Q T$ interval. After the patient had rested in the supine position for $10 \mathrm{~min}$, the QT interval of three consecutive sinus beats was measured to the end of the $T$ wave on electrocardiogram tracings taken at a paper speed of $50 \mathrm{~mm} / \mathrm{s}$. The QT interval corrected (QTc) for the cardiac cycle length was calculated according to Bazett's formula QTc $=\mathrm{QT} /(\sqrt{\mathrm{RR}})$ [21] and reported in milliseconds. The upper limit for a normal QTc interval was taken as $440 \mathrm{~ms}$ [22].

\section{Statistical analysis}

Data are expressed as mean value $\pm 1 \mathrm{SD}$. Differences in the groups were compared using the Mann-Whitney U-test. The parameter-free Spearman's rank test was used for correlation coefficients. For the myocardial uptake score of MIBG (MU score) the Wilcoxon-matched-pairs signed rank test was used. A $p$-value of less than 0.05 was considered significant.

\section{Results}

Scintigraphically the diabetic patients did not display myocardial perfusion abnormalities $\left({ }^{99} \mathrm{~m}\right.$ Tc-MIBI study). All the patients had an MU score of $\leq 2$.

All the control subjects demonstrated a normal uniform homogeneous global myocardial uptake of ${ }^{123}$ I-MIBG (MU score $\leq 2$ ). In contrast, in the CANnegative group a normal homogeneous global tracer uptake was observed in only six diabetic patients $(27 \%)$, whereas 16 diabetic patients $(73 \%)$ in this group demonstrated a reduced tracer uptake (MU score $>2$ ). Furthermore, only one diabetic patient in the CAN-positive group (5\%) presented a normal homogeneous uptake and 19 diabetic patients $(95 \%)$ a reduced tracer uptake. A severe reduction of uptake or no uptake (MU score of $\geq 5$ ) was observed in ten diabetic patients of the CAN-positive group $(50 \%)$ and in four diabetic patients of the CAN-negative group (18\%). The mean global myocardial uptake was significantly smaller in the CANpositive group than in the CAN-negative group (MU score $4.4 \pm 1.1$ vs $3.4 \pm 1.3, p<0.01$; Fig. 3 ).

Figure 2 depicts the typical pattern of the distribution of myocardial uptake in a diabetic patient with ECG-based cardiac autonomic neuropathy in comparison to a control subject.

Regional differences of the myocardial uptake between the two groups were found in the lateral and posterior myocardial region. The mean lateral and posterior myocardial uptake was smaller in the CAN-positive group than in the CAN-negative group (MU score $4.4 \pm 1.3$ vs 3.1 $\pm 1.6, p<0.01$ and $5.5 \pm 0.8$ vs $4.0 \pm 1.5, p<0.001$; Fig. 4 and Fig. 5 ). Ten diabetic patients in the CAN-positive group $(50 \%)$ and four diabetic patients in the CAN-negative group $(18 \%)$ presented an MU score of $\geq 5$ in the lateral region. In the posterior region an MU score of $\geq 5$ was observed in 19 diabetic patients of the CAN-positive group (95\%) and in 8 diabetic patients of the CAN-negative group $(36 \%)$. There was no significant difference in the uptake of the anterior and septal myocardial region between the CAN-positive group and the CAN-negative group.

The entire study group of 42 diabetic patients (20 diabetic patients with and 22 diabetic patients without ECG-based cardiac autonomic neuropathy) presented with significant differences in the regional myocardial uptake (Fig. 6 ). The posterior myocardial region demonstrated a smaller myocardial uptake than the anterior, lateral and septal myocardial region $(p<0.001, p<0.001, p=0.001)$. The anterior myocardial region showed a larger myocardial uptake than the lateral and septal region $(p<0.05, p=0.001)$. The myocardial uptake of the lateral and septal myocardial region did not differ significantly.

The coefficients of correlations between the myocardial uptake of ${ }^{123}$ I-MIBG with clinical characteris- 


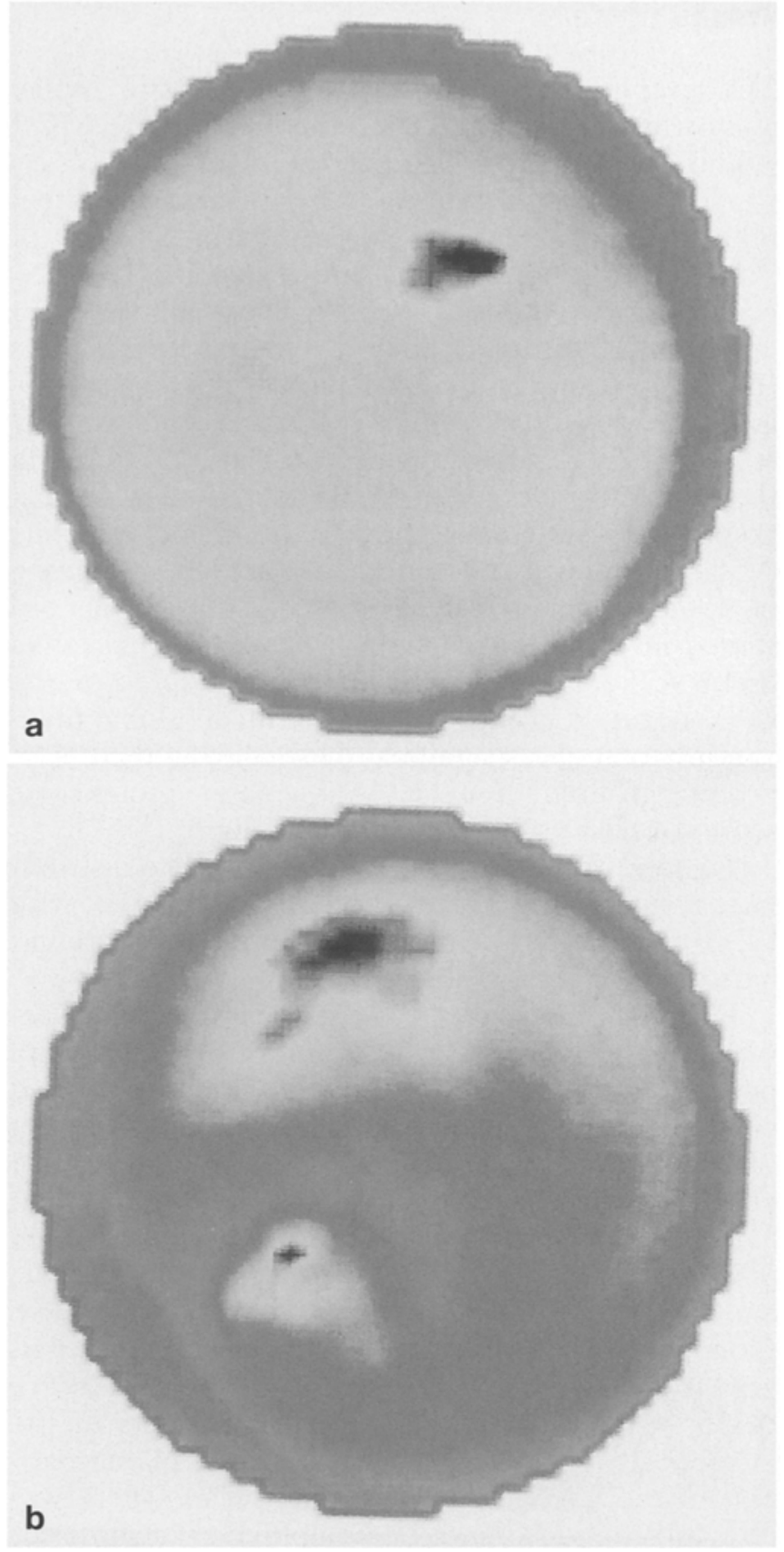

Fig. 2a, b. Polar maps of myocardial ${ }^{123}$ I-MIBG uptake. (a) Male control subject (age 37 years) with a uniform and homogeneous ${ }^{123}$ I-MIBG uptake. MU score: global 1, anterior 1, lateral 1, posterior 1 and septal 1 (b) Male IDDM patient (age 35 years, diabetes duration 16 years, $\mathrm{HbA}_{1 \mathrm{c}} 8.9 \%$ with $\mathrm{ECG}-$ based cardiac autonomic neuropathy. MU score: global 4, anterior 3 , lateral 5 , posterior 6 , septal 4

tics, cardiac reflex tests and QTc interval are shown in Table 2. The global and regional myocardial uptake did not correlate with age, body mass index, duration of diabetes and $\mathrm{HbA}_{1 \mathrm{c}}$. No correlation between the preprandial blood glucose level $(6.38 \pm 0.83 \mathrm{mmol} / 1$ in the study group) and the global and regional myocardial uptake was observed. Female diabetic patients exhibited a significantly reduced uptake in the

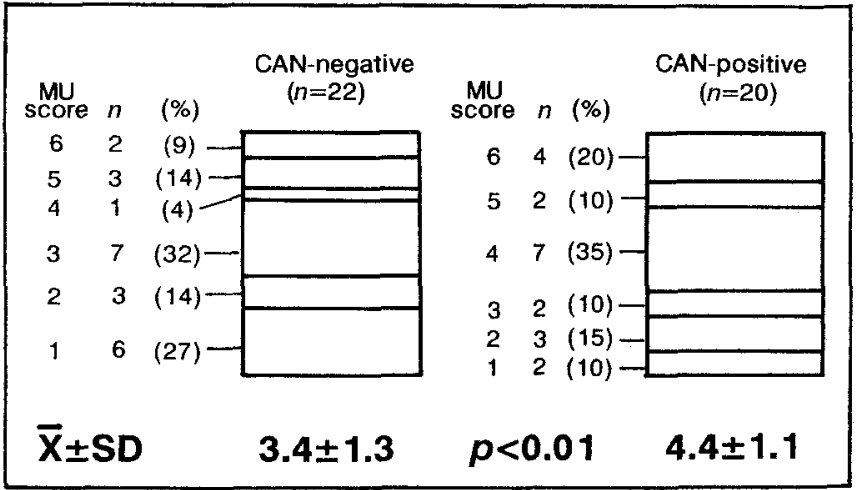

Fig.3. Comparison of global myocardial ${ }^{123} \mathrm{I}-\mathrm{MIBG}$ (MU score) between the CAN-negative and CAN-positive group

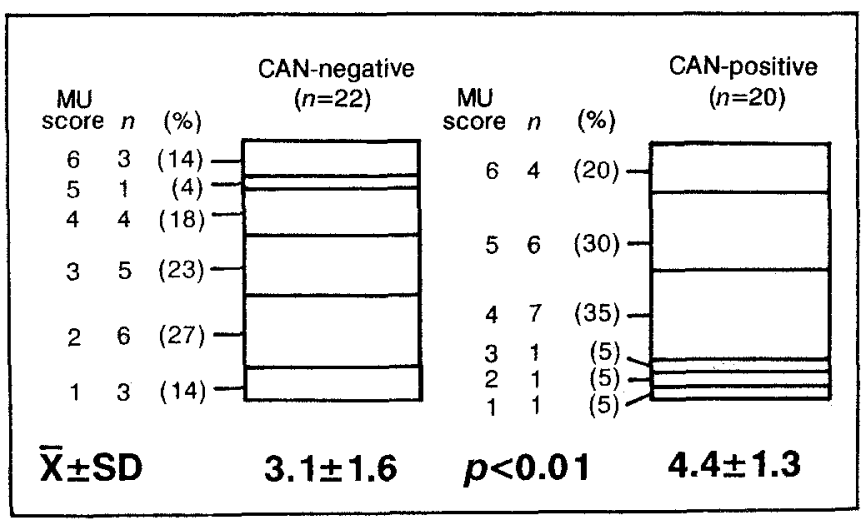

Fig. 4. Comparison of the lateral myocardial ${ }^{123} \mathrm{I}-\mathrm{MIBG}$ (MU score) between the CAN-negative and $\mathrm{CAN}$-positive group

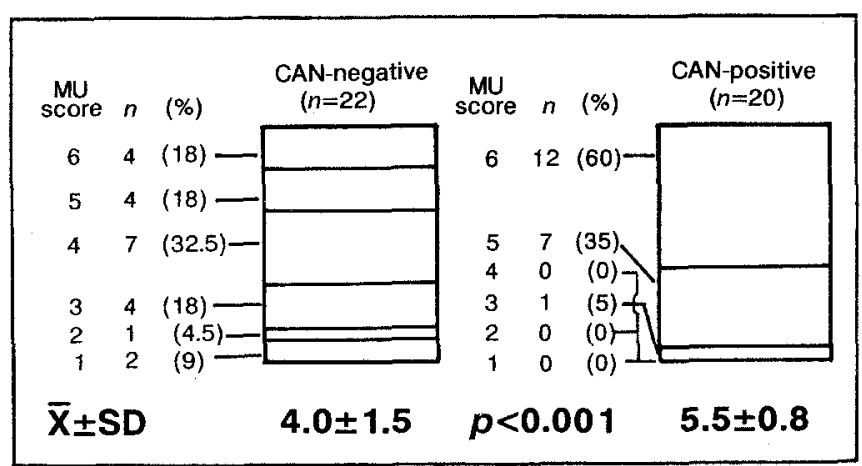

Fig. 5. Comparison of the posterior myocardial ${ }^{123} \mathrm{I}-\mathrm{MIBG}$ (MU score) between the CAN-negative and CAN-positive group

posterior myocardial region compared to male diabetic patients $(p<0.05)$. Significant correlations between the myocardial uptake of ${ }^{123} \mathrm{I}-\mathrm{MIBG}$ and cardiac reflex tests were found when compared with heart rate variation at rest and during deep breathing; the global myocardial uptake, respectively the lateral and posterior myocardial region, correlated with the index root mean squared successive difference of heart rate variation at rest $(p<0.05, p<0.01$, 


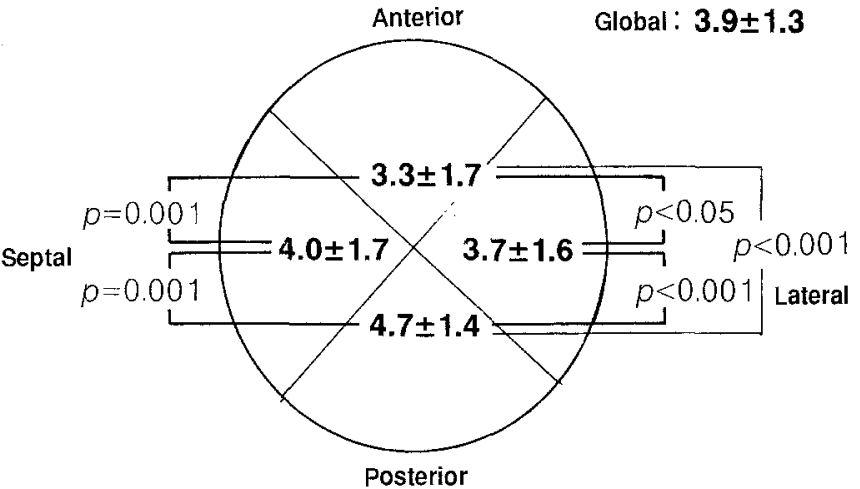

Fig. 6. Global and regional myocardial ${ }^{123}$ I-MIBG uptake in 42 IDDM patients. The posterior myocardial region demonstrated the largest and the anterior myocardial region the smallest reduction of ${ }^{123}$ I-MIBG uptake

$p<0.001)$. Furthermore, the posterior myocardial region correlated with the index coefficient of variation (CV) of heart rate variation at rest $(p<0.05)$. The posterior myocardial region correlated with four out of five indices (CV, expiration, inspiration, (E-I) difference, E-I ratio and MCR) of heart rate variation during deep breathing $(p<0.01, p<0.05, p<0.05$, $p<0.05)$. No relationship between the myocardial uptake of ${ }^{123}$ I-MIBG and the 30:15 ratio, Valsalva manoeuvre, systolic blood pressure response to standing and QTc interval was observed.

\section{Discussion}

This is the first study to assess scintigraphically global and regional differences of cardiac sympathetic dysinnervation in a larger group of clinically comparable long-term IDDM patients with and without ECGbased cardiac autonomic neuropathy. Our findings show that cardiac sympathetic dysinnervation is also present in long-term IDDM patients without ECGbased cardiac autonomic neuropathy. The existence of ECG-based cardiac autonomic neuropathy in long-term IDDM patients, is not only associated with a progression of the posterior and lateral cardiac sympathetic dysinnervation, but also with a deterioration of global cardiac sympathetic dysinnervation.

Previous studies in normal control subjects have demonstrated a uniform and homogeneous myocardial uptake of ${ }^{123}$ I-MIBG in each subject, indicating a regular pattern of myocardial sympathetic innervation in normal man $[15,23,24]$. We confirm this observation, in that all control subjects in the present investigation were found to have a uniform homogeneous uptake of ${ }^{123} \mathrm{I}-\mathrm{MIBG}$.

The high degree of reduced ${ }^{123}$ I-MIBG uptake in diabetic patients without ECG-based cardiac autonomic neuropathy suggests that cardiac sympathetic dysinnervation may be present before ECG-based cardiac autonomic neuropathy. This has also been observed in a previous study on 12 non-insulin-dependent diabetic patients with and without autonomic neuropathy [13]. Recently it has been indicated that ${ }^{123}$ I-MIBG scintigraphy might be more sensitive in detecting cardiac autonomic neuropathy in diabetic patients than heart rate variation during deep breath-

Table 2. Spearman correlation coefficients between global and regional myocardial uptake of ${ }^{123}$ I-MIBG uptake of 42 IDDM patients and clinical characteristics, cardiac reflex tests and QTc interval

\begin{tabular}{|c|c|c|c|c|c|}
\hline${ }^{123}$ I-MIBG uptake & Global & Anterior & Lateral & Posterior & Septal \\
\hline Gender & -0.26 & -0.18 & -0.19 & $-0.33^{a}$ & -0.15 \\
\hline Age & 0.11 & -0.04 & 0.08 & 0.14 & -0.08 \\
\hline Body mass index & 0.01 & 0.01 & -0.07 & 0.01 & -0.07 \\
\hline Duration of diabetes & 0.08 & -0.02 & -0.03 & -0.10 & -0.09 \\
\hline $\mathrm{HbA}_{1 \mathrm{c}}$ & 0.09 & -0.05 & 0.07 & 0.20 & 0.15 \\
\hline \multicolumn{6}{|l|}{ Heart rate variation at rest } \\
\hline \multicolumn{6}{|c|}{ Heart rate variation during deep breathing } \\
\hline $\mathrm{CV}$ & -0.25 & -0.07 & -0.24 & $-0.38^{\mathrm{b}}$ & -0.25 \\
\hline RMSSD & -0.22 & -0.05 & -0.21 & -0.29 & -0.22 \\
\hline E-I difference & -0.15 & -0.02 & -0.15 & $-0.32^{a}$ & -0.24 \\
\hline E-I ratio & -0.18 & -0.03 & -0.19 & $-0.33^{a}$ & -0.28 \\
\hline MCR & -0.18 & -0.05 & -0.18 & $-0.34^{a}$ & -0.09 \\
\hline QTc interval & -0.01 & -0.05 & -0.02 & 0.07 & 0.05 \\
\hline
\end{tabular}

Significant levels: ${ }^{a} \mathrm{p}<0.05,{ }^{\mathrm{b}} \mathrm{p}<0.01,{ }^{\mathrm{c}} \mathrm{p}<0.001$; RMSSD, Root mean squared successive difference; CV, coefficient of variation 
ing [15]. Considering the results together, it seems reasonable to conclude that the direct assessment of cardiac sympathetic innervation with the ${ }^{123}$ I-MIBG scintigraphy is methodologically superior to indirect cardiac reflex tests in detecting early cardiac dysinnervation.

A limitation of SPECT is that only relative quantitative analysis, as in exercise/redistribution myocardial scintigraphy, can be performed. Absolute quantification is not available with SPECT [25]. We obtained SPECT images after $5 \mathrm{~h}$ to allow for extraneurovesicular clearance of ${ }^{123}$ I-MIBG uptake [26]. Wash-out of ${ }^{123}$ I-MIBG was not determined. It is not clear whether enhanced wash-out reflects sympathetic tone or sympathetic denervation, or both [27]. Moreover it has been postulated that increased wash-out is non-specific [28].

The ability of sympathetic nerve endings to take up exogenously administered catecholamines is wellestablished. Reports have confirmed the existence of a high-affinity, sodium-dependent neuronal uptake mechanism (uptake-1) confined to postganglionary sympathetic nerves, which is considered to represent true intraneuronal uptake and a low-affinity extraneuronal uptake mechanism (uptake-2) $[8,29]$. Unlike noradrenaline, MIBG is metabolized neither by monoaminooxidase nor by catechol- $O$-methyltransferase $[30,31] .{ }^{123}$ I-MIBG enters the liver due to its large volume and vascularity. The subsequent rapid rates of loss from the liver may reflect the large nonneuronal pool of the radiopharmaceutical [7]. ${ }^{123}$ IMIBG is rapidly excreted in the urine in its unmetabolized form and therefore the kidneys can only be faintly visualized [32].

Sympathetic denervation has been described in infarcted myocardium with a subsequent potential for arrythmogenicity $[33,34]$. In coronary artery disease a reduction of ${ }^{123}$ I-MIBG uptake has been observed, which emphasises the high sensitivity of the sympathetic nervous system to ischaemia [35]. In the present study, however, myocardial perfusion abnormalities in the diabetic patients were excluded by means of ${ }^{99 \mathrm{~m}} \mathrm{Tc}-\mathrm{MIBI}$-scintigraphy, although there is evidence that microvascular factors such as impaired nerve blood flow play an important role in the pathogenesis of diabetic neuropathy [36]. Also, chronic hyperglycaemia has been demonstrated to be a contributing factor [37]. However, the more severe reduction of ${ }^{123} \mathrm{I}-\mathrm{MIBG}$ uptake in patients with long-term IDDM with ECG-based cardiac autonomic neuropathy compared to those without, is probably not explained by the degree of metabolic control or the duration of diabetes, since neither $\mathrm{HbA}_{1 \mathrm{c}}$ nor duration of diabetes differed between the two groups. Previous differences in metabolic control, however, cannot be excluded. Certainly, the CAN-positive group showed a more severe autonomic involvement at the time of the study.
Interestingly, the uptake of the posterior myocardial region correlated with mainly parasympathetic tests such as heart rate variation at rest and during deep breathing [3]. Tests reflecting mainly cardiac sympathetic function such as the Valsalva manoeuvre and systolic blood pressure response [3] did not correlate with myocardial ${ }^{123}$ I-MIBG uptake. However, the reasons for the relatively poor correlation between global or regional myocardial ${ }^{123}$ I-MIBG uptake and cardiac reflex tests also remain speculative. It may be hypothesized, that cardiac reflex tests do not differentiate absolutely between sympathetic and parasympathetic function.

Other reasons for a reduction of ${ }^{123} \mathrm{I}-\mathrm{MIBG}$ uptake in diabetes may be proposed. Increased levels of circulating norepinephrine could compete with ${ }^{123} \mathrm{I}$ MIBG for uptake-1. However, the competition would probably affect the heart in a homogeneous fashion and not explain regional differences as observed in the diabetic heart. Previously, no elevated venous catecholamine levels had been observed in diabetic patients with reduced myocardial ${ }^{123}$ I-MIBG uptake [13]. It is also possible that the observed reduced ${ }^{123}$ I-MIBG uptake represents a normal uptake with enhanced release of ${ }^{123}$ I-MIBG, which may not represent sympathetic denervation [38]. Nevertheless, the correlation of sympathetic denervation by electrophysiologic testing to defects on ${ }^{123} \mathrm{I}-\mathrm{MIBG}$ scintigraphy has been demonstrated in the canine model and suggests that regional ${ }^{123}$ I-MIBG defects result from regional adrenergic dysfunction [39].

The decrease of uptake of ${ }^{123} \mathrm{I}-\mathrm{MIBG}$ in the posterior myocardial region in long-term IDDM raises the possibility of arrythmogenicity. Patients with ventricular arrhythmias and absence of coronary artery disease demonstrate a similar pattern of ${ }^{123}$ I-MIBG uptake [40]. In arrythmogenic right ventricular cardiomyopathy, characterized by reduced ${ }^{123} \mathrm{I}-\mathrm{MIBG}$ uptake in the inferior and posterolateral regions, involvement of the sympathetic nervous system in arrythmogenesis has been suggested [24]. Whether the sympathetic dysinnervation of the posterior myocardial region in diabetes predisposes to arrhythmias needs to be investigated by further longitudinal studies.

Patients with idiopathic long QT syndrome have been found to demonstrate a reduced myocardial ${ }^{123}$ I-MIBG uptake indicating sympathetic dysinnervation [12]. Whether diabetes is associated with a prolonged QT interval is under debate $[41,42]$, but also a shortened QT interval in diabetes has been described [43]. We did not observe an association between a prolonged QTinterval and reduced myocardial ${ }^{123}$ I-MIBG uptake.

Our study demonstrates that global and regional cardiac sympathetic dysinnervation is common in long-term IDDM even in patients without otherwise detectable cardiac autonomic neuropathy and that the posterior myocardium is predominantly affected. 
The circumscribed reduction of ${ }^{123} \mathrm{I}-\mathrm{MIBG}$ uptake in the posterior region may have implications for predisposition to arrythmogenicity. ${ }^{123} \mathrm{I}-\mathrm{MIBG}$ scintigraphy seems to be a promising approach to further elucidate the pattern and natural history of myocardial sympathetic dysinnervation in IDDM.

\section{References}

1. Ewing DJ, Campbell IW, Clarke BF (1980) The natural history of diabetic autonomic neuropathy. Q J Med 49: 95-108

2. Sampson MJ, Wilson S, Karagiannis P, Edmonds M, Watkins PJ (1990) Progression of diabetic autonomic neuropathy over a decade in insulin-dependent diabetics. Q J Med 75: 635-646

3. Ewing DJ, Clarke BF (1982) Diagnosis and management of diabetic autonomic neuropathy. BMJ 285: 916-918

4. Ewing DJ, Boland O, Neilson JMM, Cho CG, Clarke BF (1991) Autonomic neuropathy, QT interval lengthening, and unexpected deaths in male diabetic patients. Diabetologia 34: 182-185

5. Sivieri R, Veglio M, Chinaglia A, Scaglione P, CavalloPerin P (1993) Prevalence of QT prolongation in a type 1 diabetic population and its association with autonomic neuropathy. Diabet Med 10: 920-924

6. Kline RC, Swanson DP, Wieland DM et al. (1991) Myocardial imaging in man with I-123 Meta-Iodobenzylguanidine. J Nucl Med 22: 129-132

7. Sisson JC, Shapiro B, Meyers L et al. (1987) Metaiodobenzylguanidine to map scintigraphically the adrenergic nervous system in man. $\mathrm{J}$ Nucl Med 28: 1625-1636

8. Tobes MC, Jaques S, Wieland DM, Sisson JC (1985) Effect of uptake-one inhibitors on the uptake of norepinephrine and metaiodobenzylguanidine. J Nucl Med 26: 897-907

9. Jaques S Jr, Tobes MC, Sisson, Baker JA, Wieland DM (1984) Comparison of the sodium dependency of uptake of metaiodobenzylguanidine and norepinephrine into cultured bovine adrenomedullary cell. Mol Pharm 26: 539-546

10. McGhie AI, Corbett JR, Akers MS et al. (1991) Regional cardiac adrenergic function using I-123-meta-iodobenzylguanidine tomographic imaging after acute myocardial infarction. Am J Cardiol 67: 236-242

11. Shimizu M, Sugihara N, Kita Y et al. (1992) Long-term course and cardiac sympathetic nerve activity in patients with hypertrophic cardiomyopathy. Br Heart J 67: 155160

12. Göhl K, Feistel H, Weikl A, Bachmann K, Wolf F (1991) Congenital myocardial sympathetic dysinnervation (CMSD) - a structural defect of idiopathic long QT syndrome. Pace 14: 1544-1553

13. Mäntysaari M, Kuikka J, Mustonen J et al. (1992) Noninvasive detection of cardiac sympathetic nervous dysfunction in diabetic patients using ( $\left.{ }^{123} \mathrm{I}\right)$ Metaiodobenzylguanidine. Diabetes 41: 1069-1075

14. Ziegler D, Rathmann W (1994) New aspects of diabetic cardiovascular autonomic neuropathy. Diab Stoffw 3: 410416

15. Claus D, Feistel H, Brunhölzl C, Platsch G, Neundörfer B, Wolf F (1994) Investigation of parasympathetic and sympathetic cardiac innervation in diabetic neuropathy: heart rate variation versus meta-iodo-benzylguanidine measured by single photon emission computed tomography. Clin Auton Res 4: 117-123
16. Khafagi FA, Shapiro B, Fig LM, Malette S, Sisson JC (1989) Labetol reduces iodine-131 MIBG uptake by pheochromocytoma and normal tissues. J Nucl Med 30: 481-489

17. Carvalho PA, Chiu ML, Kronauge JF, Kawamura M, Jones AG, Holman BL (1992) Subcellular distribution and analysis of technetium-99m-MIBI in isolated perfused rat hearts. J Nucl Med 33: 1516-1522

18. Chiu ML, Herman LW, Kronauge JF, Piwnica-Worms D (1992) Comparative effects of neutral dipolar compounds and lipophilic anions on technetium 99m-hexakis (2-methoxy-isobutyl isonitrile) accumulation in cultured chick ventricular myoctes. Invest Radiol 27: 1052-1058

19. Crane P, Laliberte R, Heminway S, Thoolen M, Orlandi C (1993) Effect of mitochondrial viability and metabolism on technetium-99m-sestamibi myocardial retention. Eur $\mathrm{J}$ Nucl Med 20: 20-25

20. Ziegler D, Laux G, Dannehl K et al. (1992) Assessment of cardiovascular function: age-related normal ranges and reproducibility of spectral analysis, vector analysis, and standard tests of heart rate variation and blood pressure responses. Diabet Med 9: 166-175

21. Bazett HC (1920) An analysis of the time-relations of electrocardiograms. Heart 7: 353-370

22. Schwartz PJ, Wolf S (1976) QT interval prolongation as predictor of sudden death in patients with myocardial infarction. Circulation 57: 1074-1077

23. Dae MW, O'Connell W, Botvinick EH et al. (1989) Scintigraphic assessment of regional cardiac adrenergic innervation. Circulation 79: 634-644

24. Wichter T, Hindricks G, Lerch H et al. (1994) Regional myocardial sympathetic dysinnervation in arrythmogenic right ventricular cardiomyopathy. Circulation 89: 667-683

25. Kirsch C-M, Moser E, Buell U (1985) Quantitative Auswertung von 201Tc-Myocardszintigrammen in SPECT Technik. Nuc Compact 16: 230-234

26. Nakajo M, Shimabukuro K, Yoshimura H et al. (1986) Io dine-131 metaiodobenzylguanidine intra- and extravesicular accumulation in the rat heart. J Nucl Med 27: 84-89

27. Dae MW (1994) Imaging of myocardial sympathetic innervation with metaiodobenzylguanidine. J Nucl Cardiol 1: 23-30

28. Nakajima K, Taki N, Tonami N, Hisada K (1994) Decreased 123J-MIBG uptake and increased clearance in various cardiac diseases. Nucl Med Comm 15: 317-323

29. Iversen LL (1971) Role of transmitter uptake mechanisms in synaptic neurotransmission. Br J Pharmacol 41: 571-591

30. Sisson JC, Wieland DM, Sherman P (1987) Metaiodo-benzylguanidine as an index of the adrenergic nervous system integrity and function. J Nucl Med 28: 1620-1624

31. Wellman HN, Zipes DP (1990) Cardiac sympathetic imaging with radioionated metaiodobenzylguanidine (MIBG). In: Zipes DP, Rowlands DJ (eds) Progress in cardiology 3/ 1. Lea and Febiger, Philadelphia London, pp 161-174

32. Mangner TJ, Tobes MC, Wieland DM, Sisson JC, Shapiro B (1986) Metabolism of iodine-131-metaiodobenzylguanidine in patients with metastatic pheochromocytoma. J Nucl Med 27: 37-44

33. Nishimura T, Oka H, Sago M et al. (1992) Serial assessment of denervated but viable myocardium following acute myocardial infarction in dogs using iodine-123 metaiodobenzylguanidine and thallium-201 chloride myocardial single photon emission tomography. Eur J Nucl Med 19: 25-29

34. Stanton MS, Tuli MM, Radtke NL et al. (1989) Regional sympathetic denervation after myocardial infarction in humans detected noninvasively using I-123 metaiodobenzylguanidine. J Am Coll Cardiol 14: 1519-1526 
35. Guertner C, Klepzig H, Maul FD et al. (1993) Noradrenaline depletion in patients with coronary artery disease before and after percutaneous transluminal coronary angioplasty with iodine-123 metaiodobenzylguanidine and single-photon emission tomography. Eur J Nucl Med 20: 776782

36. Tesfaye S, Malik R, Ward JD (1994) Vascular factors in diabetic neuropathy. Diabetologia 37: 847-854

37. The Diabetes Control and Complications Trial Research Group (1993) The effect of intensive treatment of diabetes on the development and progression of long-term complications in insulin dependent diabetes mellitus. $\mathrm{N}$ Eng $\mathrm{J}$ Med 329: 977-986

38. Dae MW, de Macro T, Botvinick EH et al. (1992) Scintigraphic assessment of MIBG uptake in globally denervated human and canine hearts: implications for clinical studies. J Nucl Med 33: 1444-1450

39. Minardo JD, Tuli MM, Mock BH et al. (1988) Scintigraphic and electrophysiolgic evidence of canine myocardial sym- pathetic denervation and reinnervation produced by myocardial infarction or phenol application. Circulation 78 : 1008-1019

40. Mitrani RD, Klein LS, Miles WM, Hackett FK, Burt RW, Wellman HN (1993) Regional cardiac sympathetic denervation in patients with ventricular tachycardia in the absence of coronary artery disease. J Am Coll Cardiol 22: 1344-1353

41. Ewing DJ, Neilson JMM (1990) QTinterval length and diabetic autonomic neuropathy. Diabet Med 7: 23-26

42. Sivieri R, Veglio M, Chinaglia A, Scaglione P, CavalloPerin P (1993) Prevalence of QT prolongation in a type 1 diabetic population and its association with autonomic neuropathy. Diabet Med 10: 920-924

43. Ong JJC, Sarma JSM, Venkataraman K, Levin SR, Singh BN (1993) Circadian rhythmicity of heart rate and QTc interval in diabetic autonomic neuropathy: implications for the mechanism of sudden death. Am Heart J 125: 744-752 